\title{
Pivotal role of the CCL5/CCR5 interaction for recruitment of endothelial progenitor cells in mouse wound healing
}

\author{
Yuko Ishida, ${ }^{1}$ Akihiko Kimura, ${ }^{1}$ Yumi Kuninaka,, ${ }^{1}$ Masanori Inui, ${ }^{2}$ \\ Kouji Matsushima,3,4 Naofumi Mukaida,4,5 and Toshikazu Kondo'

\begin{abstract}
1Department of Forensic Medicine and 2Department of Molecular Immunology, Institute of Advanced Medicine, Wakayama Medical University, Wakayama, Japan. ${ }^{3}$ Department of Molecular Preventive Medicine, Graduate School of Medicine, University of Tokyo, Tokyo, Japan.

${ }^{4}$ Japan Science and Technology Agency, Core Research for Evolutional Science and Technology, Tokyo, Japan.

${ }^{5}$ Division of Molecular Bioregulation, Cancer Research Institute, Kanazawa University, Kanazawa, Japan.
\end{abstract}

\begin{abstract}
BM-derived endothelial progenitor cells (EPCs) are critical and essential for neovascularization in tissue repair and tumorigenesis. EPCs migrate from BM to tissues via the bloodstream, but specific chemotactic cues have not been identified. Here we show in mice that the absence of CCR5 reduced vascular EPC accumulation and neovascularization, but not macrophage recruitment, and eventually delayed healing in wounded skin. When transferred into $\mathrm{Crr}^{-/-}$mice, $\mathrm{Crr} 5^{+/+} \mathrm{BM}$ cells, but not $\mathrm{Crr}^{-/-}$cells, accumulated in the wound site, were incorporated into the vasculature, and restored normal neovascularization. Consistent with these observations, CCL5 induced in vitro EPC migration in a CCR5-dependent manner. Moreover, expression of VEGF and TGF- $\beta$ was substantially diminished at wound sites in $\mathrm{Ccr}^{-/-}$mice, which suggests that EPCs are important not only as the progenitors of endothelial cells, but also as the source of growth factors during tissue repair. Taken together, these data identify the CCL5/CCR5 interaction as what we believe to be a novel molecular target for modulation of neovascularization and eventual tissue repair.
\end{abstract}

\section{Introduction}

Wound healing is a complex but well-orchestrated biological event, with interplay between different tissue structures and a large number of resident and infiltrating cell types $(1,2)$. The process is composed of 3 phases: inflammation, proliferation, and tissue remodeling. During the inflammatory phase, neutrophils and macrophages appear in the wounded area to phagocytose bacteria and debris. In the proliferative phase, fibroblasts produce collagen matrix, while new blood vessels invade the forming granulation tissue, and epidermal cells migrate across the wound surface to close the breach. During the remodeling phase, fibroblasts reorganize the collagen matrix and ultimately assume a myofibroblast phenotype to effect connective tissue compaction and wound contraction. The molecular mediators of wound repair have not been fully delineated, but include cytokines, chemokines, and growth factors $(1,3)$.

Chemokines are small, secreted proteins that are produced constitutively or in an inducible manner by most cell types and that induce directed cell migration $(4,5)$. Chemokines are involved in physiological and pathological processes, such as lymphoid organ development, inflammation, and tumorigenesis, because they coordinate trafficking of leukocytes, endothelial cells, fibroblasts, and other cell types, acting at specific $G$ protein-coupled receptors $(4,6)$. Several chemokines are expressed in healing wounds, and abnormal expression or activity of chemokines is associated with impaired healing (3). Although chemokine function in wound healing is thought to include recruitment of leukocytes, the precise roles of most chemokines in wound healing have not yet been addressed.

Authorship note: Yuko Ishida and Akihiko Kimura contributed equally to this work. Conflict of interest: The authors have declared that no conflict of interest exists. Citation for this article: JClin Invest. 2012;122(2):711-721. doi:10.1172/JCI43027.
Endothelial progenitor cells (EPCs) were previously identified as BM-derived endothelial precursor cells that contribute to vasculogenesis $(7,8)$. Neovascularization is essential for the survival of growing, injured, and ischemic tissue. EPCs have been presumed to be involved in a number of conditions requiring neovascularization, including peripheral vascular disease (8), myocardial ischemia (9), stroke (10), retinopathy (11), and tumor growth $(8,12)$, as well as wound healing $(8,13)$. Similar to leukocyte trafficking, EPCs are thought to mobilize from the BM into the circulation and home to injured tissues under the guidance of signals such as hypoxia, growth factors, and chemokines. However, specific molecular mechanisms that regulate the mobilization and homing of EPCs have not been defined. Here, we demonstrated that CCL5 specifically induced in vitro migration of EPCs by acting at CCR5 expressed on the cell surface. Consistently, CCR5-deficient ( $\left.\mathrm{Cr} 5^{-/-}\right)$ mice exhibited impaired neovascularization during excisional skin wound repair process, with reduced EPC homing to wound sites. Our observations suggest that the CCL5/CCR5 interaction is a major regulator of EPC homing during wound healing.

\section{Results}

Expression of CCR5 and its ligands during skin wound healing. We first examined the expression of CCR5 and its ligands, CCL3, CCL4, and CCL5, at skin wound sites in WT mice. Cor 5 mRNA and its protein were faintly detected in unwounded skin tissue. After injury, the expression of CCR5 increased at the mRNA and protein levels, reaching the maximal level at 3 days and remaining at this level through 6 days after the injury (Figure 1, A-C). At the wound sites, CCR5 protein was detected on $\mathrm{F} 4 / 80^{+}$macrophages and $\mathrm{CD}_{31}{ }^{+}$endothelial cells (Figure 1D). Concomitantly, gene expression of $\mathrm{Ccl} 3, \mathrm{Ccl} 4$, and $\mathrm{Ccl} 5$ at the wound sites started to increase at 1 day after injury. The mRNAs of $\mathrm{Ccl} 3$ and $\mathrm{Ccl} 4$ reached 
A

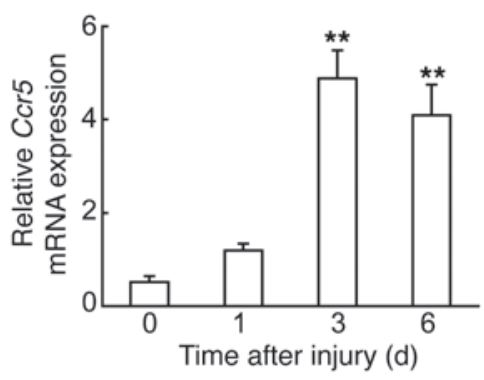

B

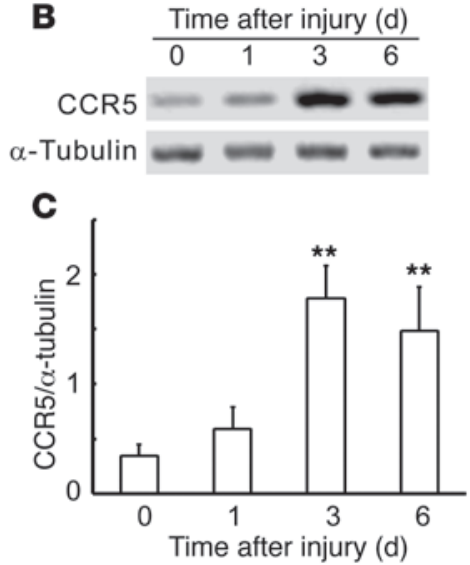

D

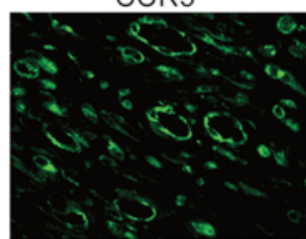

$\mathrm{F} 4 / 80$

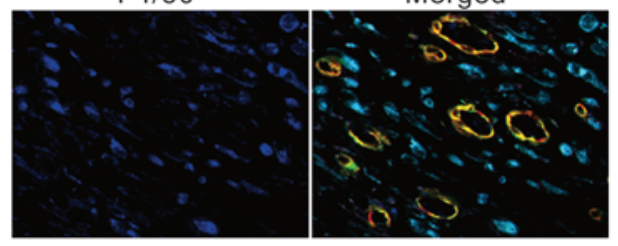

Figure 1

CCR5 expression at wound sites in WT mice. (A) Quantitative RT-PCR analysis for Ccr5 mRNA $(n=6)$. (B) Western blotting analysis of CCR5 protein expression. Representative results from 6 independent experiments are shown. (C) CCR5/ $\alpha$-tubulin ratios, calculated densitometrically ( $n=6$ independent experiments). (D) Cell types expressing CCR5 in wounded skin at day 6. Triple-color immunofluorescence images of CCR5 (green), CD31 (red), and F4/80 (blue). Representative results from 4 individual animals are shown. Original magnification, $\times 400$. All values represent mean \pm SEM. ${ }^{* *} P<0.01$ versus uninjured skin (time 0 ).

a peak at day 3, whereas $C c l 5$ mRNA reached a maximum at day 6 (Figure 2, A-C). Moreover, the protein levels of these CCR5 ligands changed in a manner similar to the mRNA results (Supplemental Figure 1; supplemental material available online with this article; doi:10.1172/JCI43027DS1). Double-color immunofluorescence analysis revealed expression of each chemokine by restricted types of cells. F4/80 macrophages expressed CCL3, CCL4, and CCL5, whereas only Ly- $6 \mathrm{G}^{+}$neutrophils expressed CCL3 (Figure 2, D-G). In contrast, no CCR5 ligands were detected in $\alpha$-SMA-positive myofibroblasts (data not shown). These observations suggested that the expression of CCR5 and its ligands was enhanced locally during skin wound healing.

Delayed wound healing with depressed neovascularization, but not macrophage recruitment, in the absence of CCR5. In WT mice, wound areas were reduced to approximately $10 \%$ at 6 days after injury, and wound closure was almost complete at 10 days (Figure 3, A and $\mathrm{B})$. On the contrary, in $\mathrm{Ccr}^{-/-}$mice, wound areas remained over $30 \%$ of the original wound area even at 6 days after injury, and wounds were not closed at 10 days (Figure 3, A and B). De novo production of collagen is essential for wound healing. Gene expression of collagen type 1 (Col1a1) was significantly reduced at day 3 and 6 in $\mathrm{Ccr}^{-/-}$versus WT mice (Figure 3C). Consistently, hydroxyproline contents, as an indicator of collagen accumulation, were significantly reduced in the wounds of $\mathrm{Crr}^{-/-}$mice compared with those of WT mice (Supplemental Figure 2). CCR5 expression by $\mathrm{F} 4 / 80^{+}$cells and CD $31^{+}$cells (Figure 1D) prompted us to explore the role of CCR5-mediated signals in macrophage recruitment and neovascularization. After wounding, macrophages were recruited to similar extents in both WT and $\mathrm{Crr}^{-/-}$mice (Figure 3, D-G). In contrast, CD31+ vessel density within the wound bed decreased in $\mathrm{Crr}^{-/-}$compared with WT mice 6 days after injury (Figure 3, H and I). Similar observations were obtained by using the antibody against another endothelial marker, VE-cadherin (Supplemental Figure 3). These observations indicated that CCR5 deficiency impaired collagen production and neovascularization, both indispensable steps for skin wound healing.
Contribution of $\mathrm{CCR}^{+} \mathrm{BM}$-derived cells to skin wound healing. Because CCR5 is expressed by BM-derived and non-BM-derived cells (5), we explored the contribution of BM-derived $\mathrm{CCR}^{+}$cells to skin wound healing by using BM chimeric mice generated from WT and $\mathrm{Crr}^{-/-}$ mice. Both WT and $\mathrm{Crr}^{-/-}$mice transplanted with $\mathrm{Crr}^{-/-}$mousederived BM cells exhibited delayed wound healing compared with the respective recipients of WT BM cells (Figure 4, A and B). Gene expression of Col1a1 and hydroxyproline content at wound sites were significantly decreased in WT and $\mathrm{Ccr}^{-/-}$mice transplanted with $\mathrm{Crr}^{-/-}$mouse-derived BM cells (Figure 4C and Supplemental Figure 4). Likewise, neovascular areas were consistently smaller in both WT and $\mathrm{Crr}^{-1-}$ mice transplanted with $\mathrm{Crr}^{-/-}$mouse-derived BM cells than in the respective WT BM recipients (Figure 4, D and E). These observations are indicative of an essential contribution of BM-derived $\mathrm{CCR}^{+}$cells to skin wound healing. We next determined the localization of BM-derived cells in skin wound sites, by intravenously injecting PKH26-labeled BM cells into lethally irradiated WT or $\mathrm{Crr}^{-/-}$mice. PKH26-labeled WT-derived BM cells were barely detected in uninjured skin sites, but accumulated in the wound sites to similar extents, whether injected into WT or $\mathrm{Ccr}^{-/-}$mice (Figure 4F). In contrast, fewer PKH26-labeled $\mathrm{Ccr5}^{-/-}$ mouse-derived BM cells accumulated at the wound bed for both recipient groups (Figure 4F). Moreover, a substantial proportion of PKH26-labeled cells expressed CD31 when they reached the skin wound site (Figure 4G), which suggested that CCR5 ${ }^{+}$BM-derived cells might contribute to neovascularization.

Reduced EPC recruitment to skin wound sites in the absence of CCR5. Evidence is accumulating in support of the crucial contribution of BM EPCs to neovascularization in damaged tissue $(8,14)$. Hence, we evaluated the dynamics of EPCs in BM, peripheral blood, and wound sites in the course of wound healing in WT and $\mathrm{Crr}^{-/-}$mice. The number of EPCs, defined as c-Kit ${ }^{+} \mathrm{Tie}-2^{+}$ cells, was constant in BM during the course of wound healing in both WT and $\mathrm{Crr}^{-/-}$mice (Figure 5, A and B) and increased in peripheral blood to a similar extent in WT and $\mathrm{Crr}^{-/-}$mice (Figure 5, C and D). In contrast, EPC numbers in wound sites were increased markedly in WT mice, but not in $\mathrm{Ccr}^{-/-}$mice (Figure 5, 

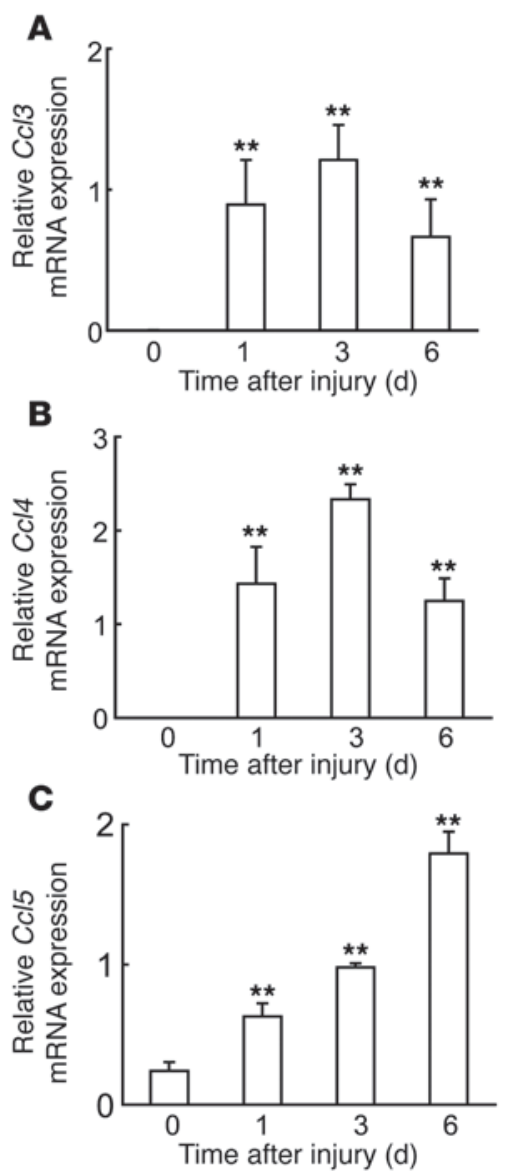

D
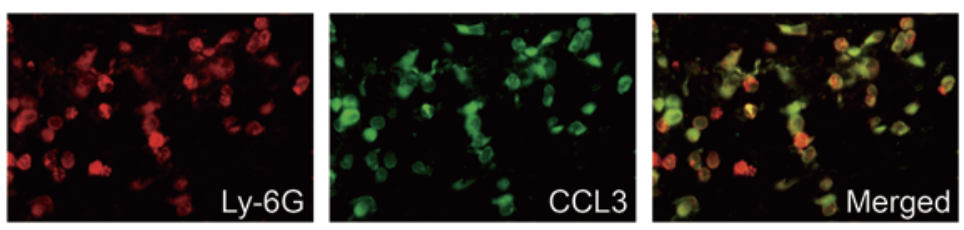

E
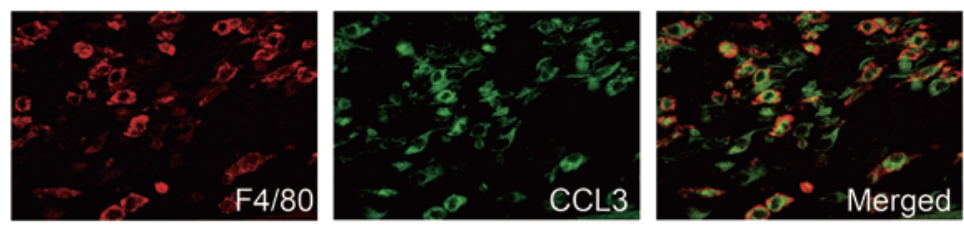

$\mathbf{F}$
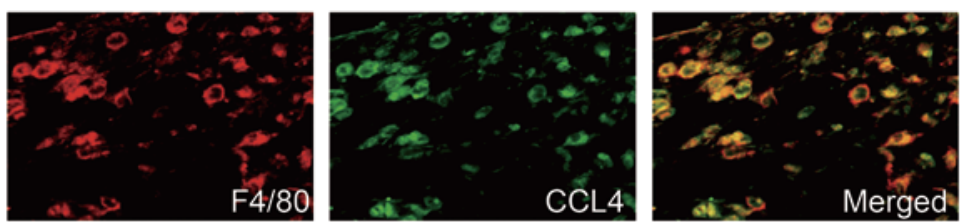

G
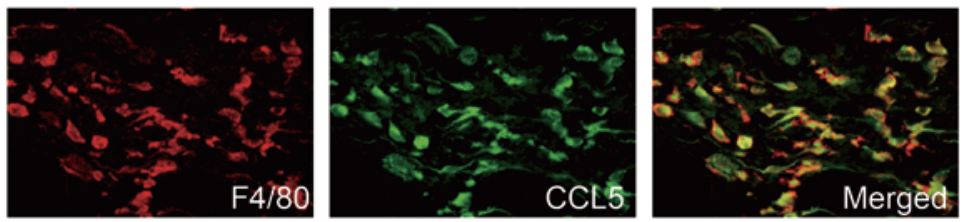

Figure 2

Expression of CCR5 ligands CCL3, CCL4, and CCL5 at wound sites in WT mice. (A-C) Quantitative RT-PCR analyses of Cc/3 (A), Cc/4 (B), and Cc/5 (C) gene expression ( $n=6$ ). (D-G) Cell types expressing CCL3 (D, day 1; E, day 6), CCL4 (F, day 6), and CCL5 (G, day 6). Representative results from 6 independent experiments are shown. Signals were merged digitally. Original magnification, $\times 400$. All values represent mean \pm SEM. ${ }^{* *} P<0.01$ versus uninjured skin (time 0 ).

E and F). Similar observations were obtained when another combination of EPC markers, CD34 and Flk-1, was used (Supplemental Figure 5). Moreover, c- $\mathrm{Kit}^{+} \mathrm{Tie}-2^{+}$cells were detected in endothelial lining cells in skin wound sites, and some of these cells expressed CCR5 (Supplemental Figure 6). Indeed, CCR5 was expressed by about $40 \%$ of $\mathrm{c}-\mathrm{Kit}^{+} \mathrm{Tie}-2^{+}$EPCs in the peripheral blood (Figure 6). Collectively, these observations indicate that CCR5-mediated signals could recruit EPCs into skin wound sites from the circulation, thereby promoting neovascularization during skin wound healing.

Essential involvement of the CCL5/CCR5 interaction in EPC migration. The expression of CCR5 ligands was significantly increased at the wound sites of WT mice after wounding (Figure 2 and Supplemental Figure 1). These observations prompted us to determine which ligand is the most potent chemoattractant for EPCs. According to the previously reported method (14-16), we obtained spindle-shaped Tie-2+ EPCs with the abilities of both Bandeiraea simplicifolia-1 (BS-1) lectin binding and Dil-acetylated LDL (AcLDL) uptake by culturing BM mononuclear cells (Supplemental Figure 7, A-C), then confirmed that a portion of the obtained Tie-2+ EPCs expressed CCR5 (Supplemental Figure 7D). CCL5 induced migration of these WT mouse-derived EPCs in a dose-dependent manner, whereas CCL3 and CCL4 lacked this activity (Figure 7, A-C). The migrating cells expressed CCR5, but not CD45 (Figure 7D), which excludes the possibility that these cells contain hematopoietic cells such as macrophages. Furthermore, EPCs derived from $\mathrm{Crr}^{-/-}$and WT mice migrated in response to VEGF and GM-CSF to a similar extent, but failed to migrate in response to CCL5 (Figure 7E). These observations indicate that CCL5 can induce EPC migration in a CCR5-dependent manner.

EPC incorporation into newly formed vessels after wounding. When PKH26-labeled cultivated WT mouse-derived EPCs were intravenously injected into WT mice immediately after wounding, the EPCs trafficked to the wound bed and were selectively incorporated into $21.8 \% \pm 3.8 \%$ of neovessels with simultaneous CD31 expression at 6 days (Figure 7F). These observations suggested that EPCs could move to the wound site to differentiate into vascular endothelial cells and eventually promote angiogenesis after the injury.

Reduced expression of VEGF and TGF- $\beta$ in mice lacking CCR5. We detected mRNAs of Vegf, Tgfb1, and Hgf in cultured EPCs (Supplemental Figure 8). Because VEGF and TGF- $\beta 1$ are indispensable for wound healing, we examined the gene expression of these factors at wound sites of WT and $\mathrm{Crr}^{-/-}$mice. Under the experimental con- 
A

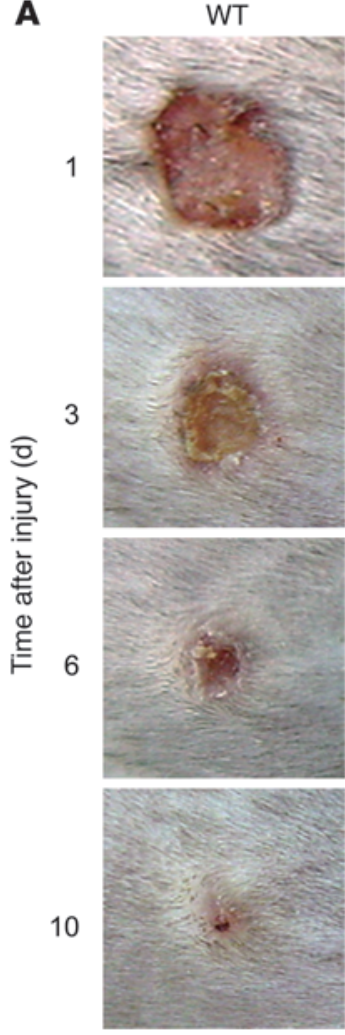

G

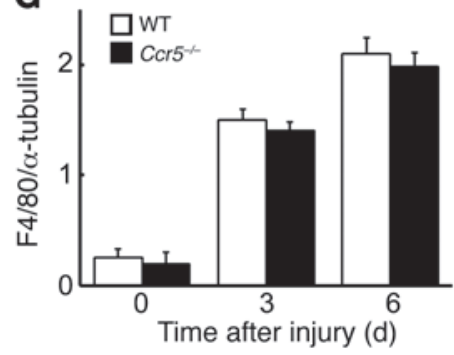

Cor5 $5^{-1}$
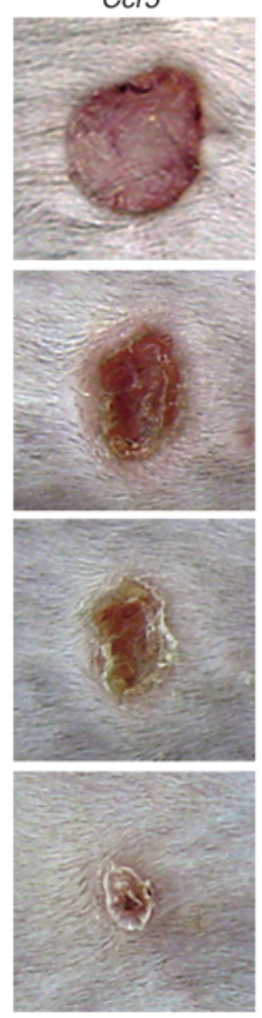

H
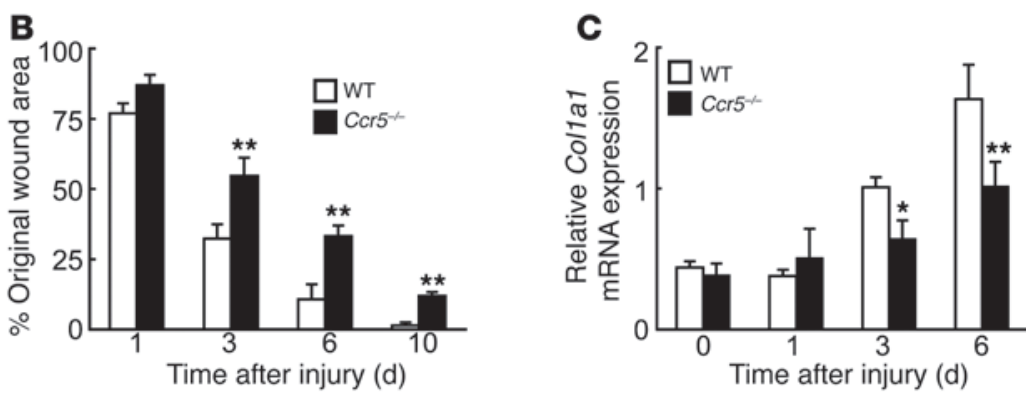

D

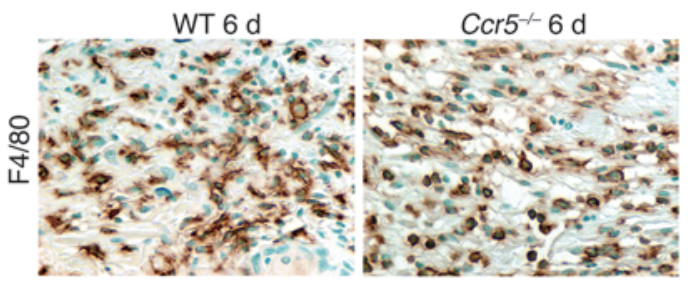

E

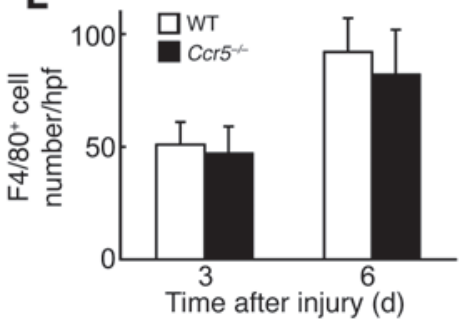

$\mathbf{F}$

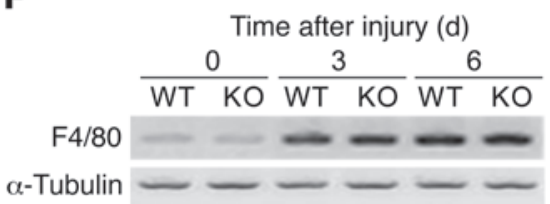

I
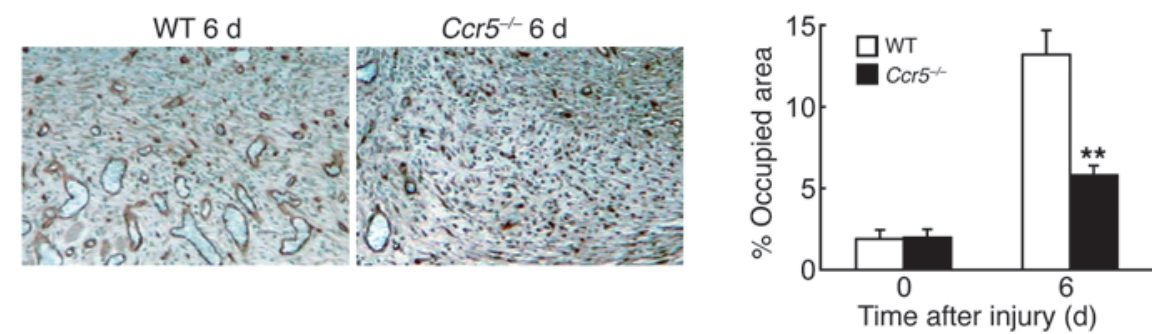

Figure 3

Skin wound healing processes in WT and $\mathrm{Ccr}^{-1-}$ mice. (A) Kinetic analysis of skin excisional wound healing. Representative results from 3 independent experiments with 4 animals per group are shown. (B) Percent wound area at each time point relative to the original wound area $(n=6)$. (C) Quantitative RT-PCR analysis for Col1a1 mRNA at wound sites $(n=6)$. (D) Immunohistochemical detection of macrophages (F4/80) at wound sites in WT and $\mathrm{Ccr}^{-1-}$ mice. Representative results from 6 independent experiments are shown. (E) Number of macrophages per high-power microscopic field (hpf, $\times 200 ; n=6$ ). (F) Western blotting analysis using anti-F4/80 Ab and anti- $\alpha$-tubulin Ab. Representative results from 6 independent experiments are shown. (G) Ratio of F4/80 to $\alpha$-tubulin $(n=6)$. (H) Neovascularization was immunohistochemically evaluated at 6 days after injury. Representative results from 4 animals are shown. (I) Vascular area, identified as percent CD31+ area with Adobe Photoshop $(n=4)$. Original magnification, $\times 400$ (D); $\times 200(\mathbf{H})$. All values represent mean \pm SEM. ${ }^{* \star} P<0.01,{ }^{\star} P<0.05$ versus WT.

ditions used, there were no significant between-strain differences in Vegf and $T g f b 1$ expression at the uninjured skin. Skin excision induced the gene expression of these growth factors in wound sites from WT mice at every time point examined, whereas $\mathrm{Ccr}^{-/-}$mice showed significant attenuation of the enhanced gene expression at 3 and 6 days after injury (Figure 8, A and B). Consistent with the quantitative RT-PCR results, the protein levels of VEGF and TGF- $\beta 1$ at the wound sites were significantly lower in $\mathrm{Ccr}^{-1-}$ mice than in WT mice (Figure 8, C and D). These observations suggested that the diminished expression of TGF- $\beta 1$ and VEGF was attributable to reduced EPC accumulation in $\mathrm{Crr}^{-/-}$mice, which would eventually result in attenuated angiogenesis and collagen production.

\section{Discussion}

Accumulating evidence indicates that involvement of the chemokine system is crucial to skin wound healing $(1,3)$. In the present study, we showed in mice that gene expression of $\operatorname{Ccr} 5$ and its ligands was upregulated at wound sites and that CCR5 protein was exclusively detected in macrophages and endothelial cells. Consistent with an important functional role, genetic disruption of Ccr5 in our model delayed skin wound healing and attenuated neovascularization, but not macrophage recruitment, whereas transfer of $\mathrm{CCR}^{+} \mathrm{BM}$ cells into $\mathrm{Ccr}^{-1-}$ mice restored normal skin wound healing and neovascularization. Moreover, the CCR5 ${ }^{+}$ $\mathrm{BM}$-derived cells were incorporated directly into the vasculature 


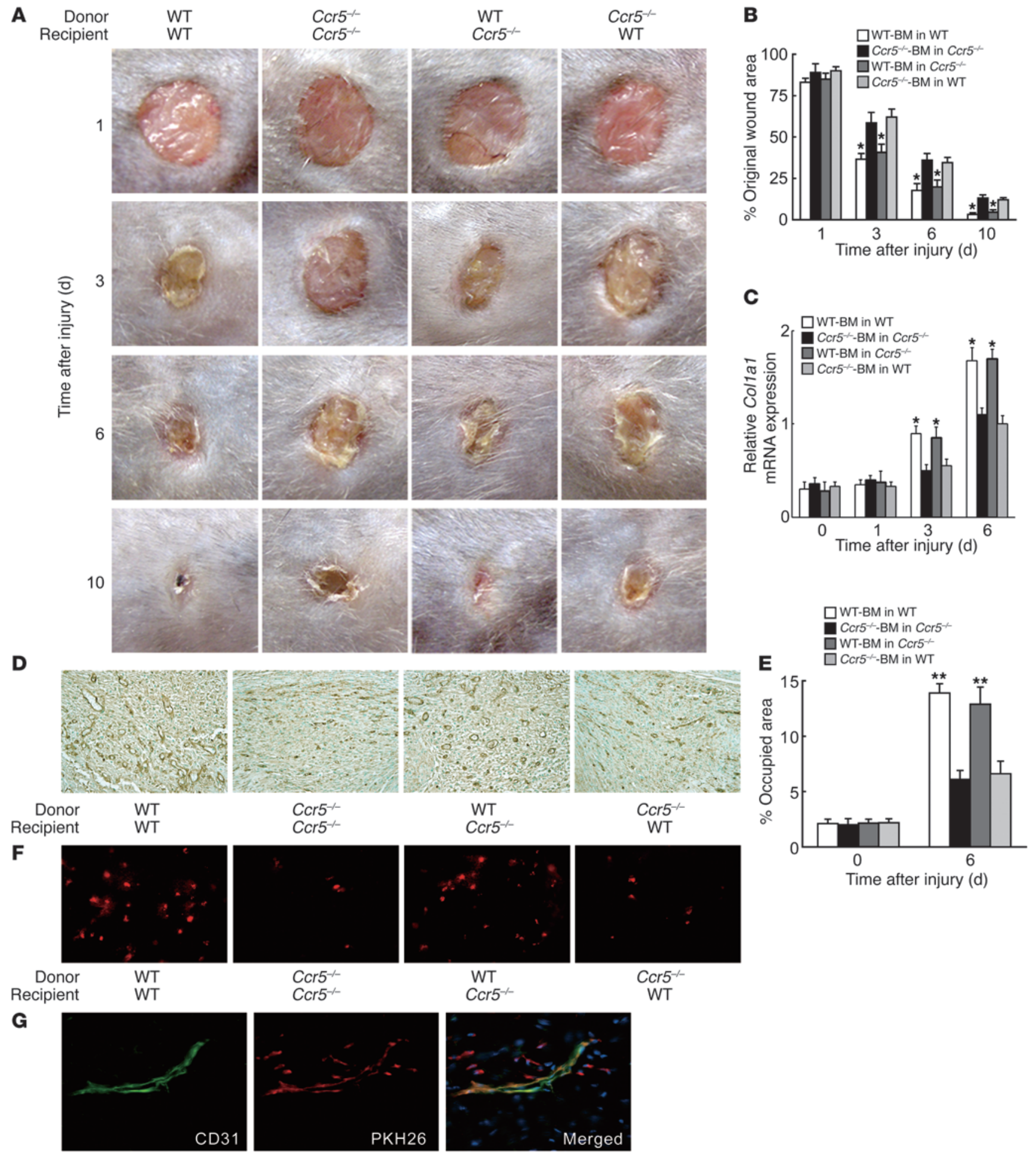

Figure 4

Effects of BM transplantation on skin wound healing of recipient mice transplanted with BM cells from WT or Ccr5 $5^{-1-}$ donors. (A) Kinetic analysis of skin excisional wound healing. Representative results from 2 independent experiments with at least 5 animals per group are shown. (B) Percent wound area at each time point relative to original area $(n=5)$. (C) Quantitative RT-PCR analysis for Col1a1 mRNA at wound sites $(n=5)$. (D) Immunohistochemical analysis with anti-CD31 mAb at 6 days after injury. Representative results from 6 individual animals are shown. (E) Vascular area, identified as percent CD31+ area with Adobe Photoshop $(n=6)$. (F) Migration of PKH26-labeled BM cells at wound sites in BM chimeric mice at day 6 after injury. Representative results from 6 individual animals are shown. (G) PKH26-labeled BM cells recruited at the wound sites colocalized with CD $31^{+}$endothelial cells. Nuclear staining is blue. Original magnification, $\times 200$ (D and F); $\times 400$ (G). All values represent mean \pm SEM. ${ }^{*} P<0.05,{ }^{* *} P<0.01$ versus respective recipients of $C c r 5^{-/-}$BM cells. 

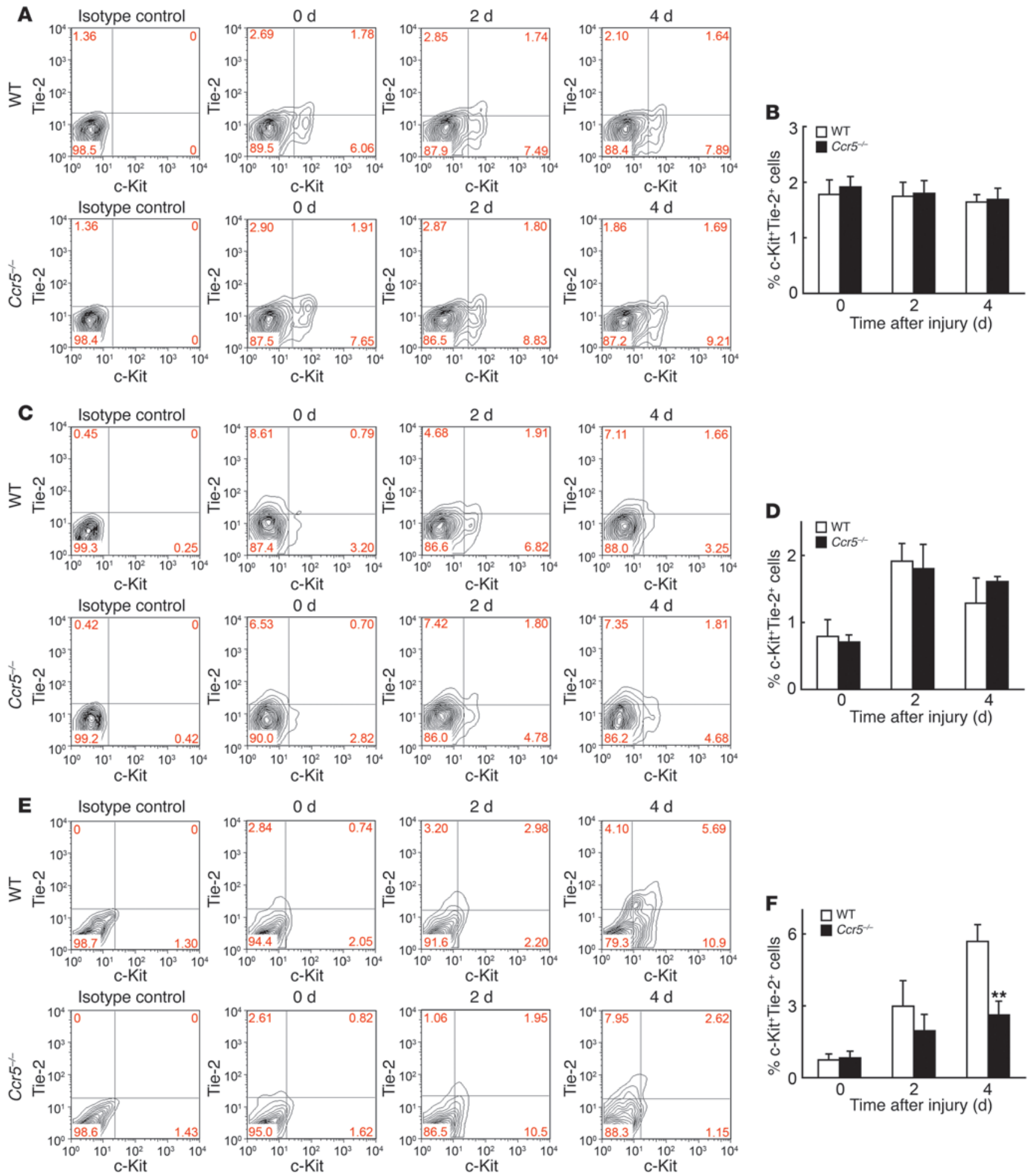

Figure 5

Quantitative evaluation of EPCs after injury. (A and B) BM, (C and $\mathbf{D})$ peripheral blood, and (E and $\mathbf{F}$ ) wound sites were analyzed to determine c-Kit ${ }^{+}$Tie-2 ${ }^{+}$EPCs. (A, C, and E) Flow cytometric analysis. Representative results from 6 independent experiments are shown. Numbers within plots denote percent cells in the respective gates. (B, D, and F) Percent C-Kit ${ }^{+} \mathrm{Tie}-2^{+}$EPCs. Values represent mean \pm SEM $(n=6) .{ }^{* *} P<0.01$ versus WT. 
A

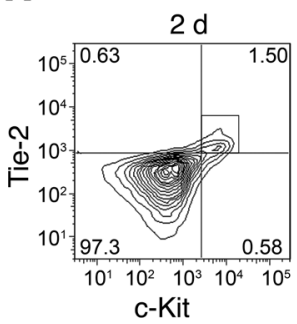

B

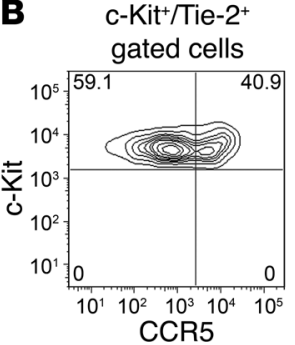

in wound sites. These observations indicated that $\mathrm{CCR}^{+} \mathrm{BM}$ cells - except for macrophages - could contribute to neovascularization, an indispensable event for sustaining the newly formed granulation tissue (2).

Asahara and colleagues identified circulating EPCs as the key cell type contributing to neovascularization (7), and subsequent studies revealed that BM-derived EPCs are essential for the tissue repair process in ischemia-induced damage of the limb, kidney, and heart $(9,17,18)$. BM-derived EPCs can also be incorporated into newly

\section{Figure 6}

Flow cytometric analysis of CCR5 $5^{+}$EPCs among c-Kit+Tie-2+ EPCs in peripheral blood cells. (A) C-Kit ${ }^{+} \mathrm{Tie}^{2}{ }^{+} \mathrm{EPCs}$ and (B) CCR5 ${ }^{+} \mathrm{EPCs}$ among $\mathrm{C}-\mathrm{Kit}^{+} \mathrm{Tie}-\mathrm{2}^{+} \mathrm{EPCs}$. Representative results from 6 individual animals are shown. Numerals within plots denote percent cells in the respective gates.

formed capillaries in granulation tissue, thereby promoting neovascularization during wound healing $(16,19)$. We also observed EPC recruitment to wound sites in WT mice and demonstrated that this process was CCR5 dependent. These observations suggest that CCR5-mediated signals can regulate EPC recruitment from BM to skin wound sites. This notion was further supported by our observations that EPCs expressed CCR5 (Figure 6 and Supplemental Figures 7 and 9).

BM-derived EPCs can be incorporated to newly formed vessels through multiple steps, including sensing the ischemic signal from the remote tissue, releasing EPCs from the BM niche into circulation, homing circulating EPCs to the target tissues, integrating EPCs into blood vessels, and in situ differentiation/maturation of
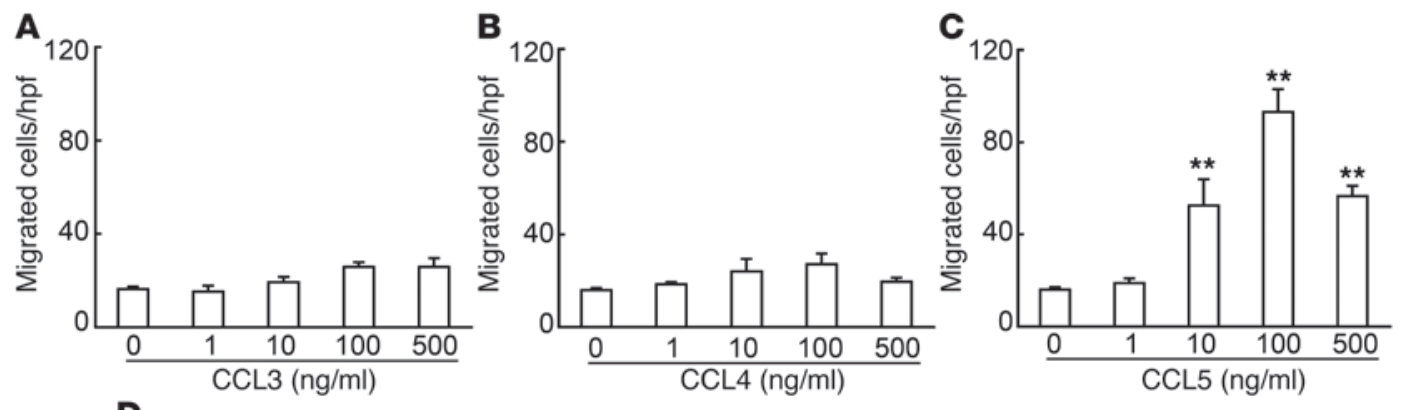

D
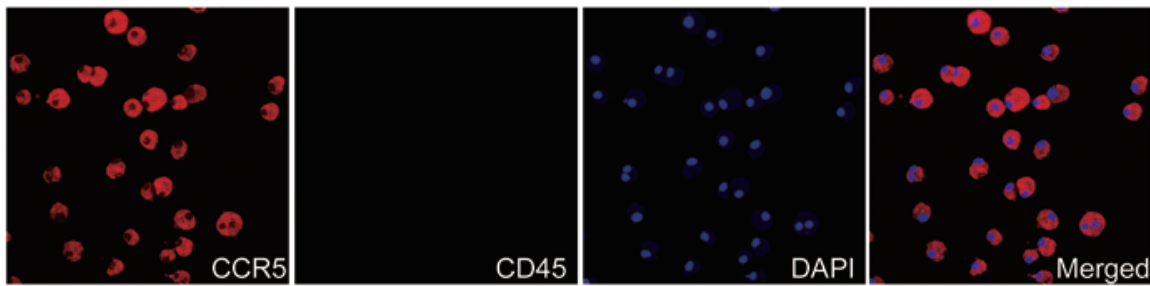

E
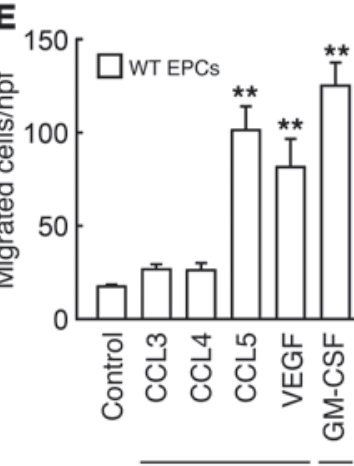

$\mathbf{F}$
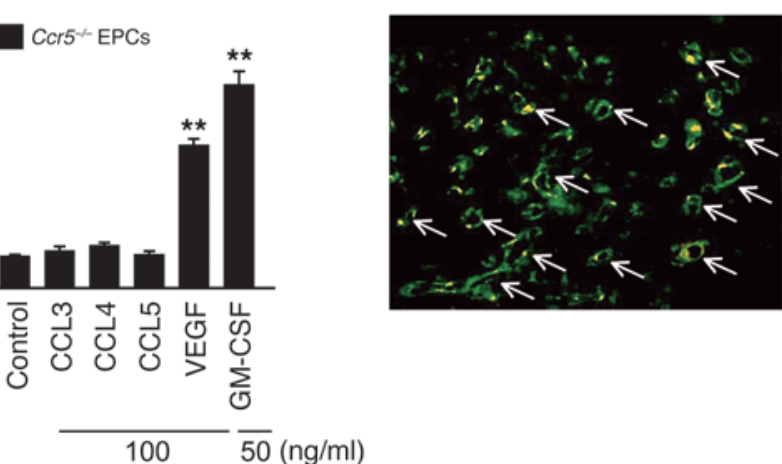

Figure 7

In vitro migratory response of EPCs to CC chemokines. (A-C) Effect of (A) CCL3, (B) CCL4, and (C) CCL5 on the migratory response of WT mouse-derived EPCs. Results are from 4 independent experiments. (D) CCL5-migrated EPCs expressed CCR5 (red), but not CD45 (green). Nuclei were stained with DAPI (blue). (E) Migratory activity in WT and Ccr5 $5^{-/-}$EPCs in response to CCR5 ligands, VEGF, and GM-CSF. Results are from 4 independent experiments. (F) EPCs were incorporated into wound capillaries in vivo (arrows). Yellow color indicates that EPCs (PKH26) were colocalized with CD31 (FITC). Representative results from 6 independent experiments are shown. Original magnification, $\times 200$ (D); $\times 100(\mathbf{F})$. All values represent mean \pm SEM. ${ }^{* \star} P<0.01$ versus medium alone (control). 

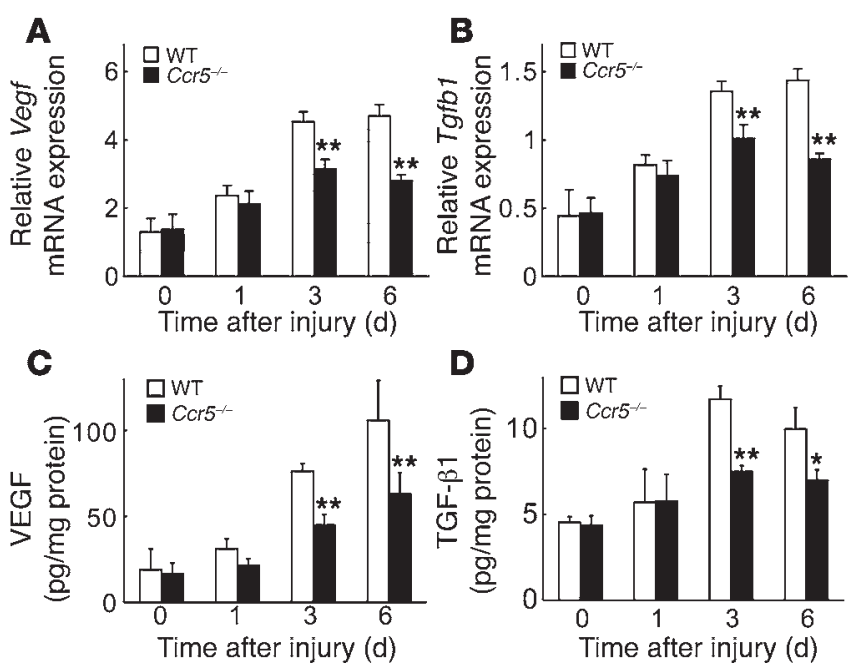

D

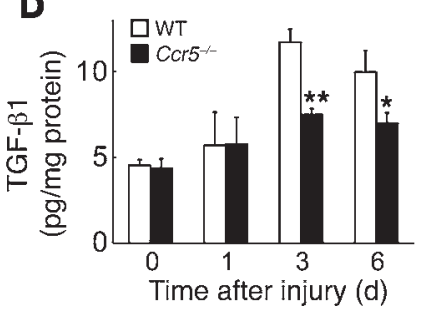

Figure 8

Decreased VEGF and TGF- $\beta 1$ expression at wound sites in $\mathrm{Ccr} 5^{-/-}$ versus WT mice. VEGF and TGF- $\beta 1$ expression was determined by quantitative RT-PCR (A, Vegf mRNA; B, Tgfb1 mRNA) and ELISA (C, VEGF protein; D, TGF- $\beta 1$ protein). Values represent mean \pm SEM $(n=6) .{ }^{* \star} P<0.01$ versus WT.

EPCs into mature and functional endothelial cells $(17,20)$. Tissue ischemia is presumed to be the strongest stimulus for EPC mobilization from the BM to the circulation $(14,17)$. Moreover, EPC mobilization can be augmented by various cytokines, including G-CSF, GM-CSF VEGF, and placental growth factor (15, 21-23). Wounding increased the number of circulating EPCs to a similar extent in WT and $\mathrm{Crr5}^{-/-}$mice. Thus, CCR5-mediated signals may not regulate EPC mobilization from the BM into the circulation, but can control the EPC-homing step and/or later steps.

It still remains unclear how circulating EPCs are recruited to target tissue. The chemokine system is essential for recruitment of various cells $(3,24)$. In line with previous studies (25-27), we detected gene expression of several chemokine receptors, such as Cor1, Ccr2, Ccr5, and Cxcr4, in cultured EPCs (Supplemental Figure 9). Several previously reported lines of evidence indicated that the CXCL12/CXCR4 interaction is partially responsible for EPC migration $(26,28)$. Spring and colleagues revealed that EPCs home into tumor, but do not mobilize from BM, in a CCR2- and/or CCR5-dependent manner (25). Thus, CXCR4-, CCR2-, and CCR5mediated signaling can regulate EPC homing into the injured sites. However, we failed to detect a significant difference in the expression of the CXCR4 ligand, CXCL12, or the CCR2 ligand, CCL2, at the wound sites between WT and $\mathrm{Crr}^{-/-}$mice (Supplemental Figure 10). Thus, CXCR4- or CCR2-mediated signals can have few roles in EPC homing in this skin excisional wound healing process. It is more plausible that CCR5-mediated signals can directly control EPC homing from the circulation to wound sites, thereby contributing to neovascularization and subsequent wound healing.

Redundancy exists in the interaction among several chemokines and chemokine receptors. CCR5 is used by 3 specific ligands, CCL3, CCL4, and CCL5, and these ligands can use another chemokine receptor, CCR1 (29). Chemokines bind to heptahelical G protein-coupled receptors $(4,6)$. Each chemokine receptor uses multiple additional and distinct signaling pathways, such as Jak/Stat, MAPK, and PI3K, depending on the ligand and cell type

involved (30). Moreover, chemokine signals can also be modulated through receptor dimerization, phosphorylation, desensitization, and internalization (31). These complexities can result in the previously observed different ligand efficacy in the CC chemokine/CCR5/CCR1 axis (32-35). In line with these observations, CCL5, but not CCL3 or CCL4, induced EPC migration in vitro to an extent similar to that of VEGF or GM-CSF. Moreover, given the previous observations that genetic deletion of CCL3 or CCR1 has no effect on neovascularization and subsequent wound healing $(36,37)$, these observations suggest that the interaction between CCL5 and CCR5 is specifically involved in EPC homing from the circulation to injured sites.

Several reports, including our present study, have demonstrated that EPCs are incorporated into mature endothelial cells, but their contribution was estimated to be only $12 \%-25 \%$ of endothelial cells in newly formed vessels in animal models $(27,38-42)$. However, impaired EPC recruitment to injured or tumor sites causes severe angiogenesis inhibition $(12,43)$, consistent with our present observations. EPCs can release VEGF, HGF, G-CSF, GM-CSF, PDGF-B, and other growth factors that are important for the neovascularization process (43-45). We detected mRNA of Vegf, Tgfb1, and Hgf in cultured EPCs (Supplemental Figure 8). Actually, in $\mathrm{Ccr}^{-/-}$mice, the gene expression and protein levels of VEGF and TGF- $\beta 1$ were significantly diminished at the wound sites. Thus, in addition to the differentiation to endothelial cells, we conclude that migrated EPCs simultaneously produce these growth factors to guide the accumulation of endothelial cells and to promote collagen production and neovascularization, eventually accelerating wound healing.

In summary, we have shown that CCR5-deficient mice display reduced EPC recruitment and subsequent attenuated neovascularization and collagen production in skin wounds, eventually resulting in delayed wound healing. If CCR5 plays a similar role in humans, our data predict that individuals lacking CCR5 as a result of homozygous inheritance of the complete loss-of-func-

\section{Table 1}

Primers used for real-time RT-PCR

\begin{tabular}{ll} 
Primer & \multicolumn{1}{c}{$\begin{array}{c}\text { Sequence } \\
\text { Ccl2 forward }\end{array}$} \\
Ccl2 reverse & 5'-GCATCCACGTGTTGGCTCA-3' \\
Ccl3 forward & 5'-CTCCAGCCTACTCATTGGGATCA-3' \\
Ccl3 reverse & 5'-TGAACCAGCAGCCTTTGCTC-3' \\
Ccl4 forward & 5'-AGGCATTCAGTTCCAGGTCAGTG-3' \\
Ccl4 reverse & 5'-CCATGAAGCTCTGCGTGTCTG-3' \\
Ccl5 forward & 5'-GGCTTGGAGCAAAGACTGCTG-3' \\
Ccl5 reverse & 5'-AGATCTCTGCAGCTGCCCTCA-3' \\
Cxcl12 forward & 5'-GGAGCACTTGCTGCTGGTGTAG-3' \\
Cxcl12 reverse & 5'-GACTTCTACCCTCGCCAAGTTCC-3' \\
Ccr5 forward & 5'-ACACTGAACCCATCGCTGCTTA-3' \\
Ccr5 reverse & 5'-GGTTCCTGAAAGCGGTGTAAATA-3' \\
Vegf forward & 5'-CTGTTGGCAGTCAGGCACATC-3' \\
Vegf reverse & 5'-GAGGATGTCCTCACTCGGATG-3' \\
Tgfb1 forward & 5'-GTCGTGTTTCTGGAAGTGAGCAA-3' \\
Tgfb1 reverse & 5'-GTGTGGAGCAACATGTGGAACTCTA-3' \\
Col1a1 forward & 5'-TTGGTTCAGCCACTGCCGTA-3' \\
Col1a1 reverse & 5'-GCTGAGATGGCAACAGGGCTA-3' \\
Actb forward & 5'-TTGATGGACGGGATGAGCA-3' \\
Actb reverse & 5'-CATCCGTAAAGACCTCTATGCCAAC-3'
\end{tabular}

Primer

Ccl3 forward

Ccl3 reverse

Cxcl12 forward

Cxcl12 reverse

Vegf reverse

Tgfb1 forward

Tgfb1 revers

Actb reverse 
tion allele $\operatorname{Cr} 5 \Delta 32$ may exhibit impaired wound healing. This allele is common among individuals of mixed European descent, with a frequency of $9.2 \%(46-48)$. A second clinical implication of our work is that the CCL5/CCR5 interaction should be considered as a potential molecular target for modulation of neovascularization. Although the properties of EPCs may warrant their use for inducing neovascularization in various clinical situations, the major obstacle for this treatment at present is the difficulty in obtaining a large number of EPCs. Exploitation of CCL5/CCR5 signaling could overcome this obstacle by promoting migration of a sufficient number of EPCs to the site where neovascularization is required.

\section{Methods}

Reagents and Abs. Recombinant murine CCL3, CCL4, CCL5, VEGF, and GM-CSF were purchased from R\&D Systems. The following mAbs and polyclonal Abs (pAbs) were used for immunohistochemical and immunofluorescence analyses: rat anti-mouse Ly-6G mAb, rat anti-mouse $\mathrm{CD} 31 \mathrm{mAb}$, and FITC-conjugated rat anti-mouse c-Kit mAb (BD Biosciences); rat anti-mouse F4/80 mAb (AbD Serotec); rabbit anti-F4/80 pAbs (Abcam); mouse anti- $\alpha$-SMA mAb (DakoCytomation); goat anti-CCL3/ MIP-1 $\alpha$ pAbs (GeneTex); rabbit anti-CCL4/MIP-1 $\beta$ pAbs (Torrey Pines Biolabs); goat anti-CCL5/RANTES pAbs, rabbit anti-Tie-2 pAbs, goat anti-CCR5 pAbs, and mouse anti- $\alpha$-tubulin $\mathrm{mAb}$ (SantaCruz Biotechnology). For flow cytometric analysis, the following Abs were commercially obtained: PE-conjugated rat anti-mouse Flk-1 mAb, FITC-conjugated rat anti-mouse $\mathrm{CD} 34 \mathrm{mAb}$, and FITC-conjugated rat anti-mouse c-Kit mAb (BD Biosciences); PE-conjugated rat anti-mouse Tie- $2 \mathrm{mAb}$ and biotinconjugated hamster anti-CCR5 mAb (eBioscience).

Animals. Specific pathogen-free 8- to 10-week-old male C57BL/6 mice were obtained from Japan SLC and designated as WT mice in this study. Homozygous $\mathrm{Crr}^{-/-}$mice, generated as described previously (49), were backcrossed onto C57BL/ 6 mice for more than 10 generations. All animals were housed individually in cages under specific pathogen-free conditions during the experiments. Age- and sex-matched mice were used for the experiments.

Experimental wound preparation and analysis. Full-thickness skin wounds were created in the dorsal skin under sterile conditions as described previously (50). Briefly, mice were anesthetized with i.p. administration of pentobarbital (50 $\mu \mathrm{g} / \mathrm{g}$ weight). After shaving and cleaning with $70 \%$ ethanol, the dorsal skin was picked up at the midline and punched through 2 layers of skin with a sterile disposable biopsy punch $(4 \mathrm{~mm}$ diameter; Kai Industries), generating 1 wound on each side of the midline. The same procedure was repeated 3 times, generating 6 wounds. Each wound site was digitally photographed at the indicated time points after injury, and wound areas were determined on photographs using Adobe Photoshop (version 7.0; Adobe Systems). Changes in wound area was expressed as the percentage of the initial wound area. In some experiments, wounds and their surrounding areas, including the scab and epithelial margins, were taken at the indicated time intervals with a sterile disposable $8-\mathrm{mm}$ diameter biopsy punch.

Generation of BM chimeric mice. The following BM chimeric mice were prepared: male $\mathrm{C} c r 5^{-/-} \mathrm{BM} \rightarrow$ female WT mice, male WT BM $\rightarrow$ female $\mathrm{Ccr}^{-/-}$ mice, male WT BM $\rightarrow$ female WT mice, and male $\mathrm{Ccr}^{-1-} \mathrm{BM} \rightarrow$ female $\mathrm{Crr}^{-/-}$mice (51). BM cells were collected from the femurs of donor mice by aspiration and flushing. Recipient mice were irradiated at 12 Gy and then intravenously received $5 \times 10^{6} \mathrm{BM}$ cells from donor mice in a volume of $200 \mu$ sterile PBS under anesthesia. Mice were subsequently housed in sterilized microisolator cages and fed normal chow and autoclaved hyperchlorinated water for 6 weeks. To verify successful engraftment and recon- stitution of BM in the transplanted mice, genomic DNA was isolated from peripheral blood and tail tissues of each chimeric mouse 6 weeks after BM transplantation with NucleoSpin Tissue Kit (MACHEREY-NAGEL). Then, we performed PCR to detect the Sry gene contained in the Y chromosome (forward primer, 5'-TTGCCTCAACAAAA-3'; reverse primer, 5'-AAACTGCTGCTTCTGCTGGT- $3^{\prime}$ ). The amplified PCR products were fractionated on $2 \%$ agarose gel and visualized by ethidium bromide staining. After durable BM engraftment was confirmed, full-thickness skin wound was made in the recipient mice as described above. In some experiments, BM cells $\left(5 \times 10^{6}\right)$ labeled with PKH26 (Sigma-Aldrich) were intravenously injected to recipient mice immediately after whole-body irradiation to delineate the trafficking of BM-derived cells.

Histopathological and immunohistochemical analyses. Wound specimens were fixed in $4 \%$ formaldehyde buffered with PBS and then embedded with paraffin to prepare sections. The sections were stained with $\mathrm{H} \& \mathrm{E}$ for histopathological analysis or were subjected to immunohistochemical analysis to evaluate neovascularization, as described previously (50). Histopathological and immunohistochemical findings were evaluated by 2 examiners without any prior knowledge of the experimental procedures.

Double- or triple-color immunofluorescence analysis. Double- or triple-color immunofluorescence analysis was conducted in order to identify the localization of CCR5, CCL3, CCL4, and CCL5 proteins as described previously (50). In some experiments, tissue samples of skin wounds were fixed in $4 \%$ PBS-buffered paraformaldehyde, to make $6-\mu \mathrm{m}$-thick sections. Thereafter, the sections were incubated with FITC-conjugated anti-mouse c-Kit Ab, anti-Tie-2, and anti-CCR5 Ab, as described previously (18).

Measurement of bydroxyproline content. Hydroxyproline content was measured as an index of collagen accumulation at the wound sites as described previously (50). Hydroxyproline content was calculated by comparison to standards (Sigma-Aldrich) and expressed as the amount per wound in micrograms.

Western blotting analysis. At the indicated time intervals after injury, wound samples were homogenized with a lysis buffer $(20 \mathrm{mM}$ Tris-HCl, $\mathrm{pH} 7.6 ; 150 \mathrm{mM} \mathrm{NaCl}$; $1 \%$ Triton; and $1 \mathrm{mM}$ EDTA) containing Complete Protease Inhibitor Cocktail (Roche) and centrifuged to obtain lysates. The lysates $(30 \mu \mathrm{g})$ were electrophoresed in a 10\% SDS-polyacrylamide gel and transferred onto PVDF membrane (50). The membrane was then incubated with anti-F4/80 $\mathrm{mAb}$, anti-CCR5 $\mathrm{Ab}$, or anti- $\alpha$-tubulin $\mathrm{mAb}$. After the incubation of secondary Abs, the immune complexes were visualized using ECL System according to the manufacturer's instructions.

Mononuclear cell isolation and flow cytometric analysis. Mononuclear cells were isolated from $\mathrm{BM}$, peripheral blood, or wound tissue homogenates as described previously $(15,18)$. Contaminated red blood cells were hemolyzed using ammonium chloride solution (IMGENEX). The resulting single-cell suspensions were incubated with the combination of PE-conjugated rat anti-mouse Tie- $2 \mathrm{mAb}$ and FITC-conjugated rat anti-mouse c-Kit $\mathrm{mAb}$ or that of PE-conjugated rat anti-mouse Flk-1 mAb and FITC-conjugated rat anti-mouse $\mathrm{CD} 34 \mathrm{mAb}$ for 20 minutes on ice. Isotype-matched control immunoglobulins were used to detect nonspecific binding of immunoglobulin. The stained cells were analyzed on a FACS Calibur flow cytometer (BD), and the obtained data were analyzed using CellQuest Pro software (BD Biosciences). Tie- $2^{+} \mathrm{c}-\mathrm{Kit}^{+}$or $\mathrm{CD} 34^{+} \mathrm{Flk}-1^{+}$double-positive cells within the myeloid mononuclear cell population were enumerated as EPCs. In some experiments, the cells were stained with the combination of anti-Tie-2, anti-c-Kit, and anti-CCR5 antibodies to determine the CCR5 ${ }^{+}$cells among EPCs.

EPC culture assay. Whole BM cells were harvested by flushing femurs and tibias with PBS, and mononuclear cells were isolated by Histopaque1083 (Sigma-Aldrich) density gradient centrifugation. Isolated BM mononuclear cells were cultured in EBM-2 BulletKit medium (Clonetics; Lonza) supplemented with $5 \%$ FBS, antibiotics, and growth factors (EPC 
medium) on fibronectin-coated 6-well plates or 4-well glass slides coated with fibronectin (BD Biosciences). 4-7 days after culture, attached spindle-shaped cells were confirmed to express the markers of EPCs, AcLDL (Invitrogen) and BS-1 lectin $(14,15)$. Cultured EPCs were subjected to immunohistochemical, and RT-PCR analyses.

RT-PCR analysis. Total RNA was extracted from whole tissue or cultured EPCs using ISOGEN (Nippon Gene), according to the manufacturer's instructions. Total RNA was reverse transcribed to cDNA using PrimeScript Reverse Transcriptase (Takara Bio) with oligo(dT) ${ }_{15}$ primers. Firststrand cDNA was synthesized from $3 \mu \mathrm{g}$ total RNA with oligo(dT) ${ }_{15}$ primers using PrimeScript Reverse Transcriptase (Takara Bio). As described previously (52), real-time PCR was carried out using SYBR Premix Ex Taq II (Takara Bio) with specific primer sets (Table 1). Primers were purchased from Takara Bio. Amplification and detection of mRNA were performed using Thermal Cycler Dice Real Time System (TP800; Takara Bio) according to the manufacturer's instructions. To standardize mRNA concentrations, transcript levels of $A c t b$ were determined in parallel for each sample, and relative transcript levels were corrected by normalization based on Actb transcript levels. Moreover, the gene expression of chemokine receptors, Flk-1, and growth factors on EPCs was evaluated by RT-PCR using appropriate primer sets (Supplemental Table 1).

Migration assay of EPCs. The contents of the upper and lower chambers were separated using polycarbonate filter $(8 \mu \mathrm{m}$ pore size). Mouse EPCs cultivated for 7 days were resuspended at $5 \times 10^{4}$ cells $/ 100 \mu \mathrm{l}$ in EBM-2 medium supplemented with $0.5 \%$ BSA and were seeded in the upper compartment. Recombinant mouse CCL3, CCL4, CCL5, VEGF (100 $\mu \mathrm{g} / \mathrm{ml})$, or GM-CSF $(50 \mu \mathrm{g} / \mathrm{ml})$ was added to the lower compartment (15). The chambers were incubated at $37^{\circ} \mathrm{C}, 5 \% \mathrm{CO}_{2}$, for 12 hours. The cells migrated into the lower chamber were counted manually in 5 random high-power fields (magnification, $\times 100$ ) per well. All groups were studied at least in triplicate. In some experiments, migrated cells were subjected to immunostaining with anti-CCR5 $\mathrm{Ab}$ and anti-CD45 $\mathrm{Ab}$.

EPC adoptive transfer. PKH26-labeled EPCs $\left(5 \times 10^{5}\right)$ in $100 \mu \mathrm{l}$ EBM-2 media without growth factors were administered to WT mice via the tail vein immediately after wounding. To evaluate the accumulation of labeled EPCs at wounded sites, wound samples were removed 5 days later, and fro- zen sections of wound samples were immunostained with anti-CD31 mAb, followed by the observation using fluorescence microscopy.

ELISA. Wound tissues were homogenized with $0.5 \mathrm{ml}$ lysis buffer $(10 \mathrm{mM}$ PBS; $0.1 \%$ SDS; $1 \%$ Nonidet P-40; and 5 mM EDTA) containing Complete Protease Inhibitor Mixture (Roche Diagnostics). The homogenates were centrifuged at $18,000 \mathrm{~g}$ for 15 minutes. The supernatants were subjected to ELISA. CCL2, CCL3, CCL4, CCL5, CXCL12, TGF- $\beta 1$, and VEGF protein levels were measured with commercially available ELISA kits (Quantikine M; $\mathrm{R} \& \mathrm{D})$ according to the manufacturer's recommendation. The detection limits for each method were as follows: CCL2, >2 pg/ml; CCL3, >10 pg/ml; CCL4, $>7$ pg/ml; CCL5, >5 pg/ml; CXCL12, > 40 pg/ml; VEGF, >3 pg/ml; TGF- $\beta 1$, $>7 \mathrm{pg} / \mathrm{ml}$. Total protein in the supernatant was measured with a commercial kit (BCA Protein Assay Kit; Pierce). The data were expressed as cytokine or growth factor $(\mathrm{pg} / \mathrm{ml})$ relative to total protein $(\mathrm{mg} / \mathrm{ml})$ for each sample.

Statistics. Means and SEMs were calculated for all parameters determined in this study. Statistical significance was evaluated using 1-way ANOVA with post-hoc testing with Scheffe's $F$ multiple comparison test or Mann-Whitney's $U$ test. A $P$ value less than 0.05 was accepted as statistically significant.

Study approval. All animal experiments were approved by the Committee on Animal Care and Use at Wakayama Medical University.

\section{Acknowledgments}

This work was supported in part by Grants-in-Aids for Scientific Research (A) (grant 20249040, to T. Kondo) and for Young Scientists (A) (grant 20689015, to Y. Ishida) from the Ministry of Education, Culture, Science, and Technology of Japan and by Research Grant on Priority Areas (to T. Kondo) from Wakayama Medical University.

Received for publication August 31, 2011, and accepted in revised form November 16, 2011.

Address correspondence to: Toshikazu Kondo, Department of Forensic Medicine, Wakayama Medical University, 811-1 Kimiidera, Wakayama 641-8509, Japan. Phone: 81.73.441.0641; Fax: 81.73.441.0641; E-mail: kondot@wakayama-med.ac.jp.
1. Werner S, Grose R. Regulation of wound healing by growth factors and cytokines. Physiol Rev . 2003;83(3):835-870.

2. Singer AJ, Clark RA. Cutaneous wound healing. NEngl J Med. 1999;341(10):738-746.

3. Gillitzer R, Goebeler M. Chemokines in cutaneous wound healing. J Leukoc Biol. 2001;69(4):513-521.

4. Charo IF, Ransohoff RM. The many roles of chemokines and chemokine receptors in inflammation. NEngl J Med. 2006;354(6):610-621.

5 . Horuk R. Chemokine receptors. Cytokine Growth Factor Rev. 2001;12(4):313-335.

6. Gerard C, Rollins BJ. Chemokines and disease. Nat Immunol. 2001;2(2):108-115.

7. Asahara $\mathrm{T}$, et al. Isolation of putative progenitor endothelial cells for angiogenesis. Science. 1997; 275(5302):964-967.

8. Asahara $\mathrm{T}$, et al. Bone marrow origin of endothelial progenitor cells responsible for postnatal vasculogenesis in physiological and pathological neovascularization. Circ Res. 1999;85(3):221-228.

9. Kawamoto A, et al. Therapeutic potential of ex vivo expanded endothelial progenitor cells for myocardial ischemia. Circulation. 2001;103(5):634-637.

10. Zhang ZG, Zhang L, Jiang Q, Chopp M. Bone marrow-derived endothelial progenitor cells participate in cerebral neovascularization after focal cerebral ischemia in the adult mouse. Circ Res. 2002; 90(3):284-288

11. Grant MB, et al. Adult hematopoietic stem cells pro- vide functional hemangioblast activity during retinal neovascularization. Nat Med. 2002;8(6):607-612.

12. Lyden D, et al. Impaired recruitment of bone-marrow-derived endothelial and hematopoietic precursor cells blocks tumor angiogenesis and growth. Nat Med. 2001;7(11):1194-1201.

13. Liu ZJ, Velazquez OC. Hyperoxia, endothelial progenitor cell mobilization, and diabetic wound healing. Antioxid Redox Signal. 2008;10(11):1869-1882.

14. Yoon $\mathrm{CH}$, et al. Intercellular adhesion molecule-1 is upregulated in ischemic muscle, which mediates trafficking of endothelial progenitor cells. Arterioscler Thromb Vasc Biol. 2006;26(5):1066-1072.

15. Asahara T, et al. VEGF contributes to postnatal neovascularization by mobilizing bone marrowderived endothelial progenitor cells. EMBOJ. 1999; 18(14):3964-3972.

16. Galiano RD, et al. Topical vascular endothelial growth factor accelerates diabetic wound healing through increased angiogenesis and by mobilizing and recruiting bone marrow-derived cells. Am J Pathol. 2004;164(6):1935-1947.

17. Rafii S, Lyden D. Therapeutic stem and progenitor cell transplantation for organ vascularization and regeneration. Nat Med. 2003;9(6):702-712.

18. Patschan D, Krupincza K, Patschan S, Zhang Z, Hamby C, Goligorsky MS. Dynamics of mobilization and homing of endothelial progenitor cells after acute renal ischemia: modulation by ischemic preconditioning. Am J Physiol Renal Physiol.
2006;291(1):F176-F185

19. Sivan-Loukianova E, Awad OA, Stepanovic V, Bickenbach J, Schatteman GC. CD34+ blood cells accelerate vascularization and healing of diabetic mouse skin wounds. J Vasc Res. 2003;40(4):368-377.

20. Urbich C, Dimmeler S. Endothelial progenitor cells: characterization and role in vascular biology. Circ Res. 2004;95(4):343-353.

21. Shi Q, Bhattacharya V, Hong-De Wu M, Sauvage LR. Utilizing granulocyte colony-stimulating factor to enhance vascular graft endothelialization from circulating blood cells. Ann Vasc Surg. 2002; 16(3):314-320.

22. Takahashi $\mathrm{T}$, et al. Ischemia- and cytokine-induced mobilization of bone marrow-derived endothelial progenitor cells for neovascularization. Nat Med. 1999;5(4):434-438.

23. Luttun A, Tjwa M, Carmeliet P. Placental growth factor (PlGF) and its receptor Flt-1 (VEGFR-1): novel therapeutic targets for angiogenic disorders. Ann N Y Acad Sci. 2002;979:80-93.

24. Rossi D, Zlotnik A. The biology of chemokines and their receptors. Annu Rev Immunol. 2000; 18:217-242.

25. Spring H, Schuler T, Arnold B, Hammerling GJ, Ganss R. Chemokines direct endothelial progenitors into tumor neovessels. Proc Natl Acad Sci U S A. 2005;102(50):18111-18116.

26. Walter DH, et al. Impaired CXCR4 signaling contributes to the reduced neovascularization capacity of 
endothelial progenitor cells from patients with coronary artery disease. Circ Res. 2005;97(11):1142-1151.

27. Gao D, Nolan DJ, Mellick AS, Bambino K, McDonnell K, Mittal V. Endothelial progenitor cells control the angiogenic switch in mouse lung metastasis. Science. 2008;319(5860):195-198.

28. Gallagher KA, et al. Diabetic impairments in NOmediated endothelial progenitor cell mobilization and homing are reversed by hyperoxia and SDF- $1 \alpha$. J Clin Invest. 2007;117(5):1249-1259.

29. Combadiere C, Ahuja SK, Tiffany HL, Murphy PM. Cloning and functional expression of CC CKR5, a human monocyte $\mathrm{CC}$ chemokine receptor selective for MIP-1 $\alpha$, MIP-1 $\beta$, and RANTES. J Lenkoc Biol. 1996;60(1):147-152.

30. Wong MM, Fish EN. Chemokines: attractive mediators of the immune response. Semin Immunol. 2003; 15(1):5-14.

31. Mueller A, Mahmoud NG, Strange PG. Diverse signalling by different chemokines through the chemokine receptor CCR5. Biochem Pharmacol. 2006; 72(6):739-48.

32. Oppermann M. Chemokine receptor CCR5: insights into structure, function, and regulation. Cell Signal. 2004;16(11):1201-1210.

33. Mueller A, Strange PG. CCL3, acting via the chemokine receptor CCR5, leads to independent activation of Janus kinase 2 (JAK2) and Gi proteins. FEBS Lett. 2004;570(1-3):126-132.

34. Tian Y, et al. Differential chemokine activation of CC chemokine receptor 1-regulated pathways: ligand selective activation of $\mathrm{G} \alpha_{14}$-coupled pathways. Eur J Immunol. 2004;34(3):785-795.

35. Oppermann M, Mack M, Proudfoot AE, Olbrich $\mathrm{H}$. Differential effects of CC chemokines on CC chemokine receptor 5 (CCR5) phosphorylation and identification of phosphorylation sites on the CCR5 carboxyl terminus. J Biol Chem. 1999; 274(13):8875-8885

36. Kaesler S, Bugnon P, Gao JL, Murphy PM, Goppelt $A$, Werner $S$. The chemokine receptor CCR1 is strongly up-regulated after skin injury but dispensable for wound healing. Wound Repair Regen. 2004; 12(2):193-204

37. Low QE, et al. Wound healing in MIP-1 $\alpha^{-/-}$and MCP-1 $1^{-/-}$mice. Am J Pathol. 2001;159(2):457-463.

38. Murayama T, et al. Determination of bone marrowderived endothelial progenitor cell significance in angiogenic growth factor-induced neovascularization in vivo. Exp Hematol. 2002;30(8):967-972.

39. Kawamoto A, et al. Therapeutic potential of ex vivo expanded endothelial progenitor cells for myocardial ischemia. Circulation. 2001;103(5):634-637.

40. Aicher A, et al. Essential role of endothelial nitric oxide synthase for mobilization of stem and progenitor cells. Nat Med. 2003;9(11):1370-1376.

41. Urbich C, Heeschen C, Aicher A, Dernbach E, Zeiher AM, Dimmeler S. Relevance of monocytic features for neovascularization capacity of circulating endothelial progenitor cells. Circulation. 2003; 108(20):2511-2516

42. Tepper OM, et al. Human endothelial progenitor cells from type II diabetics exhibit impaired proliferation, adhesion, and incorporation into vascular structures. Circulation. 2002;106(22):2781-2786.

43. Khakoo AY, Finkel T. Endothelial progenitor cells. Annu Rev Med. 2005;56:79-101.

44. Rehman J, Li J, Orschell CM, March KL. Peripheral blood "endothelial progenitor cells" are derived from monocyte/macrophages and secrete angiogenic growth factors. Circulation. 2003; 107(8):1164-1169.
45. Suh W, et al. Transplantation of endothelial progenitor cells accelerates dermal wound healing with increased recruitment of monocytes/macrophages and neovascularization. Stem Cells. 2005; 23(10):1571-1578.

46. Bamshad MJ, et al. A strong signature of balancing selection in the 5' cis-regulatory region of CCR5. Proc Natl Acad Sci U S A. 2002;99(16):10539-10544.

47. Blanpain C, et al. Multiple nonfunctional alleles of CCR5 are frequent in various human populations. Blood. 2000;96(5):1638-1645.

48. Mummidi S, et al. Evolution of human and nonhuman primate CC chemokine receptor 5 gene and mRNA. Potential roles for haplotype and mRNA diversity, differential haplotype-specific transcriptional activity, and altered transcription factor binding to polymorphic nucleotides in the pathogenesis of HIV-1 and simian immunodeficiency virus. J Biol Chem. 2000;275(25):18946-18961.

49. Murai M, et al. Peyer's patch is the essential site in initiating murine acute and lethal graft-versus-host reaction. Nat Immunol. 2003;4(2):154-160.

50. Ishida Y, Kondo T, Kimura A, Matsushima K, Mukaida N. Absence of IL-1 receptor antagonist impaired wound healing along with aberrant NF- $\mathrm{KB}$ activation and a reciprocal suppression of TGF- $\beta$ signal pathway. J Immunol. 2006;176(9):5598-5606.

51. Ishida Y, et al. Essential roles of the CC chemokine ligand 3-CC chemokine receptor 5 axis in bleomycin-induced pulmonary fibrosis through regulation of macrophage and fibrocyte infiltration. Am J Pathol. 2007;170(3):843-854.

52. Nosaka M, et al. Absence of IFN- $\gamma$ accelerates thrombus resolution through enhanced MMP-9 and VEGF expression in mice. J Clin Invest. 2011; 121(7):2911-2920. 